Review

\title{
A Review on the Genetic Regulation of Myogenesis and Muscle Development
}

\author{
${ }^{1,2}$ Wuyi Liu

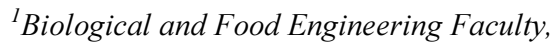 \\ Anhui Province Key Laboratory of Environmental Hormone and Reproduction, \\ Fuyang Normal University, Fuyang, China \\ ${ }^{2}$ Department of Science and Technology, Fuyang Normal University, China
}

Article history

Received: 31-12-2018

Revised: 01-01-2019

Accepted: 06-03-2019

Corresponding Author: Wuyi Liu

Faculty of Biological and

Food Engineering, Anhui

Province Key Laboratory of

Environmental Hormone and

Reproduction Fuyang Normal

University (Zip 236037), Qing

He West Road No. 100,

Fuyang 236037, China

Tel: +86-558-2596562

Fax: $+86-558-2596561$

Email:1wycau@163.com

lwycau@cau.edu.cn

\section{Introduction}

In the past 50-60 years, a great progress has been made in the genetic selection of lean meat rate, growth rate and feed efficiency of domesticated animals. The meat production levels of domesticated animals have been continuously improved and their marketing cycles have been gradually shortened, but the internal genetic regulation mechanism of meat type animals during this genetic selection is not clear yet. With the intersection and development of these research fields of modern genetics and molecular biology and developmental biology, the perceiving and understanding of myogenesis and development of animal muscle tissues gradually enter into the molecular biology level, such as the genetic control of double muscle phenomena in pigs, cattle and sheep and the genetic selection of lean muscle mass in animal development have been related and referred to candidate genes of Myostatin (firstly reported in cattle and sheep) and IGF-II (Insulin-Like Growth FactorII, firstly reported in cattle and pigs) and CLPG (Callipyge, firstly reported in sheep and goat)in many short reports and/or detailed studies (McMurtry et al., 1997; Andersson and Georges, 2004; Georges, 2007). However, the underlying genetic mechanism and interactions involved in myogenesis and development of animal muscle tissues are still unclear.
The formation and patterning of three muscle types are usually found in vertebrates, i.e. smooth, cardiac and skeletal muscles (Robson, 2017). All these three muscle types share the property that they contract to move substances around the body (smooth and cardiac) or move and stabilize the skeleton or skeletal (Robson, 2017). Specifically, the skeletal muscles are very import economic genetic traits in domestic animals (Aiello et al., 2018; Dou et al., 2018), such as the breast and leg muscles in commercial broiler chicken. In general, muscle cells of vertebrates originate from the mesoderm which is one of the early emerging cell types in initial myogenesis and development of muscle tissues. In vertebrates, the mesoderm is induced and brought about by some members of the BMP signaling molecule family. Molecular genetics and developmental biology studies showed that, myogenic regulatory factors (MRF), as the central regulatory target factors and other key regulatory molecules involved in the fate control of mesodermal cells mainly referred to many following signaling channels and/or pathways (Alfonso and Alison, 2002), i.e. Wnt signaling pathway, Shh (sonic hedgehog) signaling pathways, TGF $\beta /$ BMP (transforming growth factor beta and bone morphogenetic factor super-family) signaling pathway, Notch/Delta signaling pathway, RTK/Ras (tyrosine receptor kinase) signaling pathway 
and other various growth factor families, such as Myostatin (belonging to TGF $\beta /$ BMP signaling pathways) and MAPK kinases (belonging to RTK/Ras signaling pathways). Once the genesis of mesoderm is formed, the signaling pathways of Wnt, TGF $\beta / B M P$, FGF and IGF and other signaling molecules of these growth factor families will jointly program to determine the differentiated developmental fate of muscle cells. For instance, the interaction between the Wnt and Shh signaling pathways synergistically promotes the occurrence of muscle tissues, which is manifested as the presence or absence of muscle mass, whereas myogenic regulatory factors MyoD and Myf5 and pax-3 are actually the targets at the intersecting ends of these signaling pathways.

The process of myogenesis starts with the transcription and translation of a number of transcription factors that are expressed by some mesodermal cells. These initial transcription factors will regulate the expressions of critical genes that code for muscle specific skeletal components (e.g. myosin), cytoplasmic enzymes (e.g. creatine kinase) and other cellular proteins that are essential for the electrophysiological processes of muscle cells. Depending on the specific combination of these essential proteins, different types of muscle cells are formed and exhibit functional differentiation (e. g., the myocardial and smooth muscle and striated muscle tissues in vertebrates and the trunk muscle and viscera muscle tissues in insects and other invertebrates). It is speculated that there was a complex gene regulation network (Fig. 1) existed in the processes of animal myogenesis induced by signals from surrounding tissues
(Molkentin and Olson, 1996; Nabeshima, 1996; Palacios and Puri, 2006; Ochi and Westerfield, 2007; Maqbool and Jagla, 2007; Haye et al., 2014; Rossi et al., 2016). In particular, the gene regulatory network framework of myogenesis and muscular development was proposed in the studies of zebrafish and fruit fly (Ochi and Westerfield, 2007; Maqbool and Jagla, 2007; Haye et al., 2014). However, at present, there are still unclear and lack of research reports and/or in-depth studies for the exploration and explanation of the genetic network of various growth factors and regulators and their interactions with some specific cellular receptor and signaling pathways in the transduction and differentiation and regulation of myogenesis and muscular development in animals. Furthermore, the genetic regulation of myogenesis and muscular development and growth of animal muscle tissues is a highly complex and comprehensive successive process, which is affected by many genetic and physiological and environmental influencing factors, such as the genotype and/or haplotype and gender of animal species and/or strains (genetics), endocrine status (physiology), dietary feed level (nutrition), living and feeding environment and other internal and external factors. The effects of genetics and physiology and nutrition and other factors on the growth and development of animal muscle mass are finally implemented through the functional integration of myogenic regulatory factors and their receptors (Odle et al., 2017; Rossi et al., 2016).

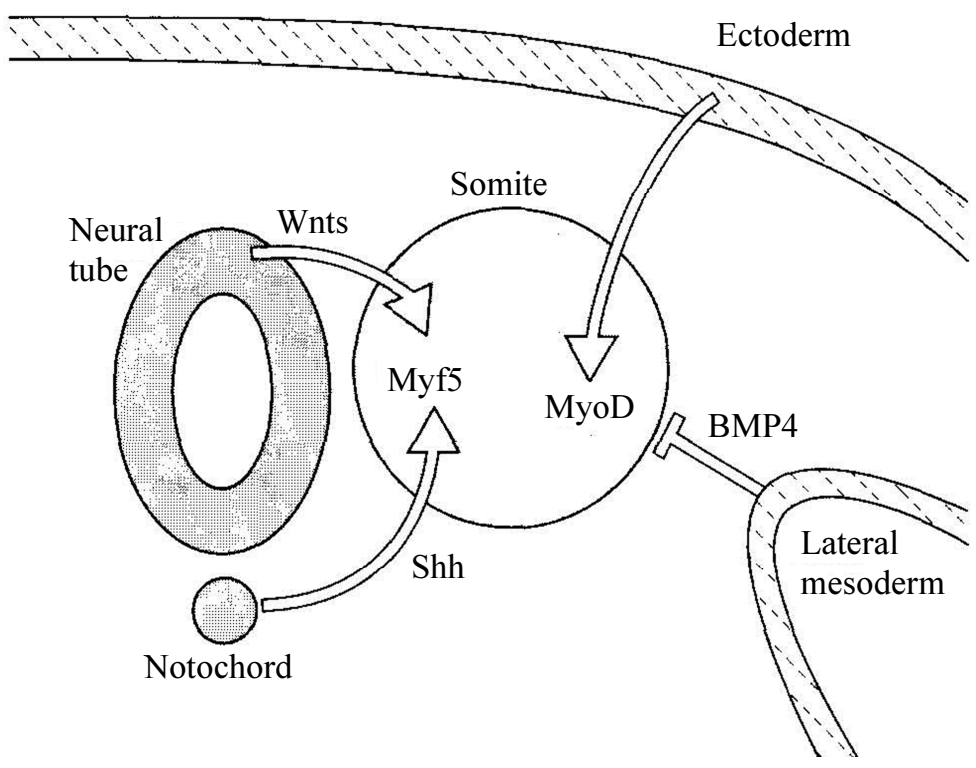

Fig. 1: A hypothetical inducting model of myogenesis in somites by signaling factors from surrounding tissues, adapted from the article by Molkentin and Olson (1996). These signaling factors act in combination along with those signaling factors from the ectoderm (top) to induce the gene expression of myogenic regulatory factors in the semite myotome. MyoD or Myf5 are expressed in proliferating myoblasts where their activity is inhibited by Cdk4 or cyclin-D 
In the recent years, muscle-specific promoters have been extensive tested and many signal transduction factors and regulatory factors have been reported as involved in muscle development research. The embryonic genetic studies of mice, birds, fish and frogs have obtained some protein and/or gene interactive networks that regulate the intergeneric myogenic signals or regulatory interactions between those signal transduction factors and regulatory factors in muscle cells, including the regulatory network and/or signaling information that drive muscle-specific gene activation in animal muscle tissues. However, our understanding of the detailed processes of myogenin and muscle development remains limited. The following parts give a full review of MRFs, Myostatin, FGF, IGF and their receptors and corresponding binding proteins in recent myogenesis and muscle development studies.

The Origin and Developmental Differentiation of Muscle Cells

The time-ordered development of muscle tissues usually begin with a variety of transcription factors expressed in some mesoderm cells. These transcription factors regulate the expression and translation of musclespecific proteins necessary for the electrophysiological processes of muscle cells, such as skeletal components and cytokines. The muscle cells (alias muscle fibers) of animals are classified into three types, i.e., red slowshrinking muscle fibers (type I), white fast-shrinking muscle fibers (type II A) and intermediate fibers (type II B), according to their different metabolic modes. Different types of muscle cells (or muscle fibers) gradually appear functionally differentiated depending on the different combinations of expressed component proteins that are transcribed and translated. Thus, the amount of muscle depends on the number and size of muscle cells (or muscle fibers). However, the number of myofibroblasts will not change once the vertebrate is born. Therefore, the growth of muscle mass and tissues in animals after birth depends mainly on the length and diameter of the postnatal muscle fibers, rather than the change in the number of muscle cells.

In practice, the process of myogenesis of muscle cells is more complicated. This process is generally divided into three stages: the first early differentiation stage, in which the mesenchymal cells undergo terminal differentiation and begin to synthesize muscle-specific proteins; the second mature stage with the musclespecific synthesis of constitutive proteins, including contractile proteins like actin and myosin, myogenic regulatory proteins like myogen, muscle cytoplasmic enzymes like creatine kinase and some special membrane proteins and their receptors; the third late differentiation stage, in which muscle cells adapt to different types of muscle fibers with different types of proteins synthesized and gradually differentiate into various muscle tissues.
The Transcription Factors and Signal Transduction Involved in the Regulation of Gene Transcriptional Activity during Myogenesis and Muscle Development

In the process of myogenesis and muscle development, the muscle cells undergo a series of phenotypic and cellular and molecular changes, resulting in the cell lineages at various stages and finally form a diversity of myogenic cells through the terminal differentiation. Ultimately, the muscle cells differentiate into multiple kinds of fast and slow muscle fibers and a variety of muscle-specific transcription factors and inhibitors have been identified and reported in these cells.

\section{The Role of Myogenic Regulatory Factors in the Cellular Determination and Differentiation of Muscle Cell}

The differentiation of myoblasts (alias myocytes) is one of the key steps in myogenesis and muscle development. In the early stages of muscle development, differentiation of the myogenic cell lineage is regulated by muscle cell-specific MRFs (myogenic regulatory factors). After an active period of cell proliferation, those myogenic cells will irrevocably withdraw from the cell cycle and fuse into multinucleated myotubes.

The myogenic regulatory factor family (alias the MyoD family) is a class of proteins containing the basic helix-loop-helix (bHLH) domains, including Myf4, Myf5, MyoD and MyoG (myogenin) that play a key role in the development of muscle cells. These transcription factors are initially found and reported in mouse and rat myoblasts and myotubes during the myogenesis and development of muscle cells. They mainly coordinate with two regulatory factors called MEF and E proteins, whereas these two proteins bind to muscle-specific genes and promote and regulate the genes' expression in embryos. The identification of myogenic regulatory factors in vertebrates stems from the research for genetic mutations that trigger the development of cultured muscle cells (Berkes and Tapscott, 2005; Rossi et al., 2016). Without these factors, muscle cells in the culture medium will not activate the program of muscle development and regeneration (Berkes and Tapscott, 2005; Rossi et al., 2016).

\section{The Discovery of Myogenic Regulatory Factors}

MyoD is the earliest MRF gene identified and analyzed among vertebrates (Choi et al., 1990; Berkes and Tapscott, 2005; Rossi et al., 2016). Studies on the induction of $\mathrm{C} 3 \mathrm{~h} 10 \mathrm{t} 1 / 2$ in embryonic cell lines using the demethylation reagent 5-heterocytoside showed that the embryonic cells underwent changes with stable genetic effects to form muscle stem cells and four members of the MRF family (MyoD, myogenin, myf-5 and myf-4) were then identified (Choi et al., 1990; Edmondson and 
Olson, 1993; Huang et al., 1998; Berkes and Tapscott, 2005; Rossi et al., 2016), in which myogenin was regarded as a key factor in the terminal differentiation of muscle stem cells into myocytes.

In vivo, each MRF is efficiently bound to a site containing CANNTG sequence (E box), which occurs in the promoter and enhancer regions of muscle-specific genes (Berkes and Tapscott, 2005; Rossi et al., 2016). Intensive expression of MRF protein in cultured nonmuscle cells can induce myogenic differentiation, but the respective efficiencies are different. However, the four MRFs showed distinct but overlapping functions in vitro. In developing mouse embryos, Myf5 is induced to express in the dorsal intermediate segment, which will develop into the trunk and intercostal muscles, followed by the expression of MyoD in the dorsal ganglion of the back which will develop into the muscles of the body wall and limbs. During the differentiation phase of muscles, MRF regulates the expression of contractile proteins and protein isoforms at different stages, allowing muscle cells to differentiate into different types of muscle fibers.

\section{Transcriptional Regulation of the Myogenic Regulatory Factor Family}

In mice, the expression of MyoD and Myf5 is a key event to the differentiation of pluripotent stem cells into muscle cell lineages (myofibril or myoblasts). Knockout or mutation of the two genes (i.e. MyoD and Myf5) in mice will result in the loss of skeletal muscle myogenic cells (Rudnicki et al., 1993; Huang et al., 1998). The absence of a mutation in either MyoD or Myf5 does not affect the normal development of the muscle, indicating a degree of redundancy in function of these two genes. Myf5 and MyoD genes control the determination of the muscle cell lineages above the axial axis (epaxial) and below the spinal axis (hypaxial) respectively (Kablar et al., 1998), while myogenin is only work in terminal differentiation of myogenic cells (Wang and Jaenisch, 1997). The functional regions (protein motifs or domains) of MyoD and Myf5 that activates a silent muscle-specific gene is the helix structure located at the C-terminal region. Therefore, the MRF4's developmental role is complex and it is regarded as a potential impacting factor in the determination and terminal differentiation of muscle cell lineages. With the sequent expression of Myf5, MRF4 was observed to be transiently expressed in mouse sarcomere at 9. 0 days (E9. 0) and faded at 11.5 days (E11.5) and re-expressed at 16. 0 days (E16.0) in mouse muscle fibers.

In addition, in the experiment of myogenin promoterMRF4 transgenic mice, the replacement of myogenin with MRF4 partially rescued the myogenesis of myogenin-/- homozygous mutant embryos (Zhu and Miller, 1997; Wang and Jaenisch, 1997), confirming that MRF4 played a role in the differentiation of muscle cells into terminal myocytes. Furthermore, the overexpression of MRF4 in embryonic stem cells (ES Cells) lacking myogenin resulted in the well-differentiated muscle fibers, whereas the over-expression of MyoD did not (Sumariwalla and Klein, 2001; Myer et al., 2001). Since the MRF4 and Myf5 genes are located in tandem sequences of DNA molecules and the experiments in vitro on the function of MRF4 are complicated, there is presently no clear conclusion of MRF4's function. Later, a series of complex Myf5 mutants have been obtained and some of these mutants can express MRF4 in the absence of Myf5 (Kassarduchossoy et al., 2004; Berkes and Tapscott, 2005; Rossi et al., 2016). Particularly, in the experiment of mice lacking both Myf5 and MyoD, the expression of MRF4 was unaffected, suggesting that MRF4 may be involved in the maturation of muscle fibers (Kassarduchossoy et al., 2004). Myogenin was then functionally inferred as distinct from Myf5 and MyoD (Rawls et al., 1995).

The previous studies mentioned above suggested the importance of MyoD and Myf5 in the establishment of muscle cell lineages. MyoD and Myf5 determine the fates of final muscle phenotypes, whereas myogenin plays a role in the terminal differentiation of muscle cells. Furthermore, MRF4 can partially promote these two regulatory processes. The functional roles of MyoD and Myf5 can be regarded as the specification of muscle cell lineages, whereas myogenin's functional role is the differentiation of muscle cells. And thus, MRF4 has some partial overlapped functions in these two aspects.

\section{The Functional Interaction between Different Regulatory Proteins or Transcription Factors}

MRF belongs to a kind of bioactive protein containing basic-helix-loop-helix (bHLH) structure of motif or domain, which is a typical type II transcription factor with tissue specific expression. MRFs can synthesize homodimers or bind to other type I factors containing bHLH structures to form heterodimers (Brennan et al., 1991; Wang and Jaenisch, 1997; Black et al., 1998; Penn et al., 2004; Berkes and Tapscott, 2005; Haye et al., 2014; Rossi et al., 2016). Particularly, type I factors mainly include protein molecules whose base regions are necessary for binding to DNA sequences, such as HEB/HTF4, E2-2/ITF-2 and E12/E47, whereas the HLH structures mediate heterodimerization between different bHLH motif containing proteins. In brief, all of the bHLH protein dimers bind to the consensus sequence CANNTG sequence of $\mathrm{E}$ box, in which each monomer in the protein dimers can recognize half of the $\mathrm{E}$ box site (i.e. CANNTG), respectively.

In addition to $\mathrm{E}$ proteins, the Id proteins belonging to the bHLH protein super-family can also bind to other bHLH containing protein or transcription factor to form heterodimers. Actually, the Id proteins have an intact bHLH domain, but they cannot bind to DNA sequences due to the lack of a critical amino acid residue in their 
basic domains, thereby inhibiting the terminal differentiation of muscle cells (Murre et al., 1989; Atchley and Fitch, 1997; Berkes and Tapscott, 2005; Haye et al., 2014; Rossi et al., 2016). Another key transcription factor interacting with bHLH motif containing proteins is the members of MEF2 family including MADS box proteins or MADS domain transcription factors identified during skeletal muscle development (Wilson-Rawls et al., 1999; Dichoso et al., 2000). Such protein factors are expressed in several mesoderm and non-mesodermal cell lineages (WilsonRawls et al., 1999; Dichoso et al., 2000). Although they do not promote muscle development by themselves, they are able to interact with bHLH motif structure containing proteins to promote the processes of myogenesis and muscle development. In the specialization of skeletal and/or cardiac muscle cells, MEF2 family members interact with $\mathrm{E}$ protein and myogenic bHLH motif containing proteins to form heterodimers, but they cannot directly from heterodimers with $\mathrm{E}$ proteins (Wilson-Rawls et al., 1999; Dichoso et al., 2000).

\section{Activators and Inhibitors in Myogenesis and Muscle Development}

As mentioned above, MRFs usually bind to the MEF proteins and other muscle-specific and/or non-musclespecific protein regulatory factors. In addition, the MRFs can act as co-activators or co-suppressors during myogenesis and muscle development to control the tissue-specific expression of related genes.

\section{Co-activators of the Muscle Tissue Specific Gene Transcription}

The cellular factors that regulate or activate MyoD and other MRFs are functionally diverse, such as the transcription factor Pax (a paired box protein), the MEF2 proteins (Lin et al., 1997; Black et al., 1998) and the members of Six family (Heanue et al., 1999) and the cell cycle regulators (Lin et al., 1997). Although most of these genes induced by MyoD were expressed at different times, chromatin immuno-precipitation experiments showed that MyoD can directly bind to corresponding regulatory elements of these genes expressed during cell differentiation of muscle tissues (Penn et al., 2004).

Members of the MRF transcription factor family, such as MyoD, Myf5, MRF4 and myogenin, were isolated by the transfection-induced developmental experiments of muscle tissues (Murre et al., 1989; Berkes and Tapscott, 2005; Haye et al., 2014; Rossi et al., 2016). Meanwhile, the same experiments also identified the regulatory protein Pax3 (Murre et al., 1989; Berkes and Tapscott, 2005), a transcription factor containing a PRD homeodomain. MyoD, Myf5 and Pax-3 are the target genes for Wnt and Shh signaling pathways. In fact, the transcription factor Pax-3 is a 479 amino acid protein encoded by the Pax gene, including two conserved DNA binding units, one consisting of a 128 amino acid amino end pairing domain and the other consisting of a 60 amino acid homeodomain. Actually, Pax-3 protein is involved in the proliferation, specialization and migration of anterior sarcomere cells in limbs. In mice and chickens, skeletal muscle cells were deduced to originate from the dermis sarcomere cells. The Pax3 gene is inferred to be expressed in a rather early dermal sarcomere, since pax-3 and the related pax-7 gene are expressed at the paraxial mesoderm before the formation of somites. Pax-3 is a key regulator of myogenesis and development of muscle cells because it activates the expression of MyoD gene in the somite and/or somatic ganglia (Rawls and Olson, 1997). The expression of Pax 3 gene in the sarcomere and/or myogenic ganglion is a synthetical result of the combination of Wnt, Shh, FGF and BMP signaling factors (Pownall et al., 2002). To explain the weak muscle developmental disorder caused by a single mutation of Myf5orMyoD or Pax3, a genetic model was proposed in mutant mice (Tajbakhsh et al., 1997), in which Myf5 and Pax3 synergistically activate MyoD expression in the myogenic segment. In the model, the regulatory effect of MyoD occurs at the downstream of the other two genes (i.e. Myf5 and Pax3) and then MyoD initiates a series of feedback regulation loops to maintain and adjust the expression levels of the three genes (Tajbakhsh et al., 1997).

\section{Co-Repressors of the Muscle Tissue Specific Gene Transcription}

In the cell culture system of muscle tissues in vitro, MyoD is expressed just prior to the activation of its target gene. Several post-translational modification models have been proposed to prevent the premature activation of MyoD, including binding to co-repressors or to other proteins that enable MyoD to detach from DNA. Many proteins identified in experiments as antagonists that inhibit muscle development directly bind to $\mathrm{E}$ proteins or MRFs, preventing them from binding to E box proteins or preventing their transcriptional activation of the muscle-specific gene promoters. Such inhibitors are themselves the proteins containing bHLH domains, including Id, Twist, MyoR and Mist-1. In addition, MyoD is also a terminal target protein for Myostatin, a key regulatory factor.

The presence of high cellular concentrations of Id proteins inhibits the activity of MRFs because Id proteins can efficiently bind to $\mathrm{E}$ box proteins to form the heterodimers that block and prevent the interaction of $\mathrm{E}$ box proteins with isolated MRFs (Benezra et al., 1990; Parrinello et al., 2001; Berkes and Tapscott, 2005). Id proteins can also form the heterodimers with MRFs, but at a very low efficiency (Benezra et al., 1990; Parrinello et al., 2001). Since Id proteins lack a base which binds to DNA sequences, the MRF/Id protein heterodimer is considered 
to be completely lacking transcriptional activity. The results and data obtained in an experiment in which Id protein was strengthened by the MyoD-E47 protein dimer, i.e. the genetic fusion of MyoD and E47 in the same peptide chain to counteract the inhibitory activity of Id protein, supports this hypothesis (Neuhold et al., 1993). Similar to Id protein, Twist is also an HLH protein which binds to $\mathrm{E}$ box protein to form a dimer and prevents the complex interactions between E box protein and MRF (Spicer et al., 1996). Unlike Id protein, Twist has one base, whereas this base binds to the base of MRF without binding to DNA sequences, preventing the mutual interaction of the $\mathrm{E}$ box region with specific MRF and its homologues on the muscle-specific promoters (Hamamori et al., 1997). Twist also inhibits the cell propagation of muscle tissues by interacting directly with the HLH binding regions of MEF2 proteins (Spicer et al., 1996).

In addition to Id and Twist, MyoR and Mist-1 and MdfI are the inhibitors of myogenesis and muscle development too. These regulatory factors contain a base region and can form a dimer with MRFs. For instance, the heterodimers MRF/MyoR and MRF/Mist-1 bind to the $\mathrm{E}$ box consensus sequence, but these dimers do not activate the transcription and translation of specific genes when linked to DNA (Lemercier et al., 1998; Lu et al., 1999). Furthermore, MdfI is actually a negative regulator of MRFs, which functions differently from other negative regulators of the proteins containing HLH structural. MdfI is a cytoplasmic protein whose function is to bind and isolate MRFs in the cytoplasm to prevent their transport into the nucleus (Chen et al., 1996).

\section{Myostatin}

Myostatin, also known as growth factor 8 (GDF8), is a member of the TGF $\beta / B M P$ super-family. The intact precursor Myostatin is a 376 amino acid long protein containing a signal peptide with an $\mathrm{N}$-terminal peptide domain and a C-terminal peptide domain of an active ligand. The C-terminal peptide domain of Myostatin includes a $12 \mathrm{kDa}$ peptide fragment. Both the unprocessed precursor Myostatin and the mature Myostatin can form a disulfide-linked dimer through this peptide fragment, but the processed mature Myostatin dimer is the only active protein form of dimerized Myostatin to bind to the cell membrane's receptors. Myostatin has been recognized as the only negative regulator of muscle growth and development in animals, since Mcpherron et al. (1997) firstly reported the mutagenic effect of Myostatin gene (or MSTN) in cattle. Myostatin has been identified as a negative regulator of skeletal muscle mass and inactivating mutations in the MSTN gene are responsible for the development of a hypermuscular phenotype, because Myostatin(-/-) mutant mice exhibit a generally and significantly increased skeletal muscle mass in which muscle atrophy occurs (Mcpherron et al., 1997; Kawada et al., 2001; Mcfarlane et al., 2006; Chelh et al., 2009), Myostatin is also regarded to play important roles in the maintenance of muscle homeostasis and regeneration (Mcpherron et al., 1997; Kawada et al., 2001; Mcfarlane et al., 2006; Chelh et al., 2009; Odle et al., 2017; Rossi et al., 2016). If Myostatin is knocked out in laboratory animals, the animal mutation would affect both the growth and development of adipose tissues and skeletal muscle tissues. The naturally occurring mutations of Myostatin gene in cattle herd, such as the homozygous mutation of 11 th $b p$ in the third exon of Myostatin gene resulted in an enlarged phenotype of double muscles in the Belgian blue cattle herd (Mcpherron et al., 1997; Mcfarlane et al., 2006). Recent researches reported in myostatin-null mice revealed that PI3K and apoptotic pathways were regarded as possible Myostatin targets and Myostatindeficiency in mice increases global gene expression at the Dlk1-Dio3 locus in the skeletal muscle (Chelh et al., 2009; Hitachi and Tsuchida, 2017; Lessard et al., 2018). Myostatin acts at key points during pre- and post-natal life of amniotes that ultimately determine the overall muscle mass of animals selected for high growth rate and muscularity (Aiello et al., 2018; Dou et al., 2018). Mutations in domestic animals have already demonstrated the impact of attenuating Myostatin activity on muscle development and a number of large animals, including cattle, sheep, goat, horse, pig, rabbit and chicken, display the double muscled phenotype or enlarged muscle mass due to mutations in the Myostatin gene (Aiello et al., 2018; Dou et al., 2018). The most recent research investigated the relations between muscle (breast and leg) myostatin mRNA expression and body and muscle growth among commercial broiler chickens (Dou et al., 2018). They found the expression of Myostatin genes was correlated negatively with body weight from day 90 to 150 and the carcass weight and muscle mass from day 90 to 150 in Avian broiler chickens (Dou et al., 2018).

In another case of mouse 9. 5-day embryos and adult skeletal muscle tissues, Myostatin is expressed preferentially in embryonic developing muscles (Dominique and Cabello, 2006). Changes in the expression amount of Myostatin have a particularly large effect on astrocytes, which may be the direct target cells of Myostatin (Cossu and Biressi, 2005; Dominique and Cabello, 2006). The Myostatin signaling pathway, which plays a inhibitory role in myogenesis and muscle development, is undergoing extensive research. A theoretical model of Myostatin signaling pathway has been proposed (Heldin et al., 1997; Franck et al., 2001; Franck and Mauviel, 2002; Dominique and Cabello, 2006; Rossi et al., 2016; Lessard et al., 2018), mainly including many cellular ligands, type I and type II receptors, Smad proteins or receptors, among which the ligands are mainly activins, Myostatin, TGF- $\beta$, BMP 
proteins and growth factors (Fig. 2). However, no receptor of Myostatin and other growth factors has been found in muscle development and regeneration so far. The most recent research shows that c-Jun N-terminal kinase (JNK) regulates muscle remodeling via myostatin/SMAD inhibition (Lessard et al., 2018). JNK is a molecular switch to active and stimulate muscle fibers to grow, resulting in increased muscle mass (Lessard et al., 2018). However, when the muscle JNK activation is suppressed and the alternative remodeling program is initiated, resulting in smaller, more oxidative muscle fibers and enhanced aerobic fitness (Lessard et al., 2018).

In addition, there is a limited and incomplete understanding of the TGF- $\beta$ signaling pathway and its members in previous studies and reports, in which most of the subjects mainly target TGF- $\beta$ and its receptors like Myostatin and there is lack of systematic research on the entire TGF- $\beta$ signaling pathway. In recent years, the molecular activities and interactions of TGF- $\beta 1$ signaling pathway in fibroblasts have been extensively studied (Heldin et al., 1997; Franck et al., 2001; Franck and Mauviel, 2002; Rossi et al., 2016; Lessard et al., 2018). In the case of skin trauma or injury or musclepathology, TGF- $\beta 1$ is firstly associated and bound with the Type II receptor (TGF- $\beta$ RII) on fibroblast membranes and then the conformation of TGF- $\beta 1$ molecule changes which can be recognized by Type I receptor (TGF- $\beta \mathrm{RI}$ ) to form the TGF- $\beta$-receptor trimer complex of Type I receptor and TGF- $\beta 1$ and Type II receptor. In this trimer complex, the Type I and Type II receptors will act on the cytoplasmic Smad proteins (i.e. Smad2 and Smad3) to activate them by phosphorylation upon the activation of TGF- $\beta$ receptors' phosphorylation, whereas the phosphorylated Smad proteins (i.e. P-Smad2 and PSmad3) form a heterotrimer with SMAD4 and transport it to the nucleus (Heldin et al., 1997; Franck et al., 2001; Franck and Mauviel, 2002; Rossi et al., 2016; Lessard et al., 2018). Then, two inhibitors of this signaling have been identified, i.e. Smad7 and Smurf1. Smad7 represses the myostatin signal by binding its $\mathrm{MH} 2$ domain to activated receptors, thus preventing recruitment and activation of R-Smads and Smurf1 is an E3 ubiquitin ligase that mediates ubiquitination and consecutive degradation of the R-Smads (Lessard et al., 2018). Thus, SMAD4 cooperates with internal transcription factors in the nucleus or independently regulates the expression of downstream target genes and promotes the synthesis of collagen and other structural components of muscle fibers.

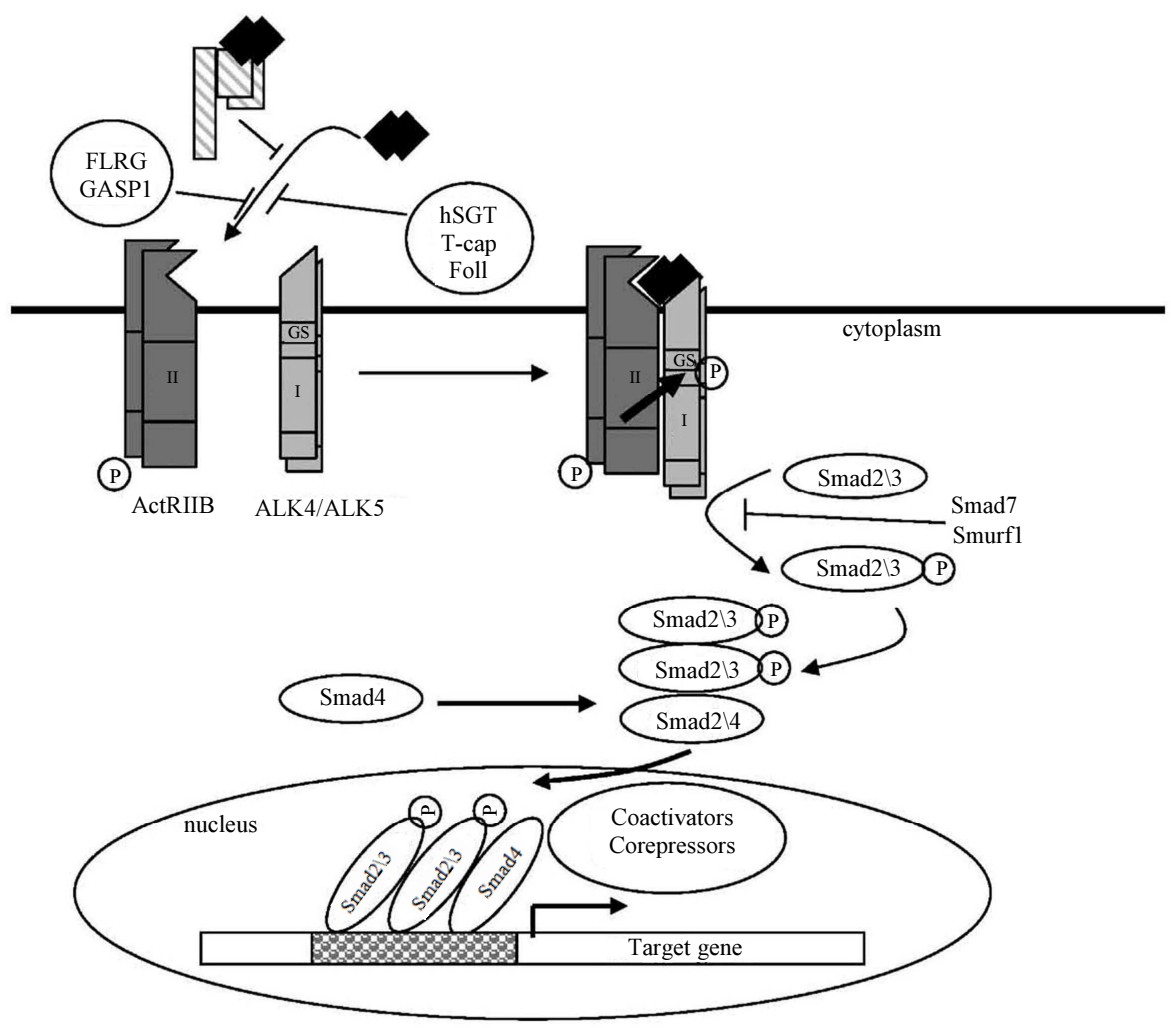

Fig. 2: A framework of Myostatin signaling pathway adapted from the article by Dominique and Cabello (2006). Those putative elements of the myostatin pathway were shown with the speculated Smad proteins and their corresponding targets and/or ligands. Myostatin can be found in serum or locally in an inactive state when bound to FLRG, GASP-1, hSGT, T-cap, or follistatin, etc. 
When the external stimulating signals of TGF- $\beta 1$ signaling pathway is over, the P-Smad proteins rapidly dephosphorylate and deactivated and the cellular Smad proteins in the cytoplasm and nucleus is rapidly metabolized by the ubiquitin-proteasome system to return to the pre-phosphorylation level before the signal action.

\section{Fibroblast Growth Factors (FGFs)}

Fibroblast Growth Factor (FGF) is a crucial signaling molecule that interacts with many proteins and receptors of the intracellular MAP kinase signaling pathway to promote various aspects of embryonic development through the FGF receptors and binding proteins (Ornitz and Itoh, 2001; Fisher et al., 2002; Itoh and Ornitz, 2011). Fibroblast growth factors and their receptors constitute a large family of polypeptide growth factors whose members are versatile (Szebenyi and Fallon, 1999; Fisher et al., 2002; Itoh and Ornitz, 2011). In the body, the expression level of FGFs is relatively constant and their roles are to respond to tissue repair and injury. FGFs will transduce their signaling mainly through four transmembrane tyrosine kinase receptors (FGFRs), i.e. FGFR1, FGFR2, FGFR3, FGFR4 (Szebenyi and Fallon, 1999). The cell culture studies of muscle tissues shown that FGFs can promote the proliferation of fibroblasts and inhibit the differentiation and myogenesis of myogenic cells. Most recent studies reported that the FGF signaling pathway directly activates the gene expression of MyoD in Xenopus (Fisher et al., 2002). Among the members of the FGF family, FGF6 (fibroblast growth factor 6) and FGF8 (fibroblast growth factor 8) and FGF24 (fibroblast growth factor 24) have been widely studied.

In the case of Fgf6(-/-) mutant mice, FGF6 was mainly expressed in the myogenic cell lines of adult muscle and developing muscle (Kästner et al., 2000; Armand et al., 2005; Mcfarlane et al., 2006; Armand et al., 2006), suggesting that it might be associated with somite formation. Indeed, the components of FGF signaling pathway were found correlated to the regeneration of adult mouse muscles. In the Fgf6(-/-) mutant mice, muscle fibers of the posterior squamous muscles of the regenerated calf showed swelling compared to those of the wild-type mice (Mynarcik et al., 1996; Kästner et al., 2000). It was surmised that Insulinlike growth factor II (IGF-II) might play a key role in the hyperplasia of this mutant mice (Mynarcik et al., 1996; McMurtry et al., 1997; Armand et al., 2005; Mcfarlane et al., 2006; Armand et al., 2006; Headey et al., 2004; Whittaker et al., 2002). In order to clarify the exact functional roles of FGF6 in muscle development and enlargement, two different laboratories produced the same FGF6 homozygous knockout individuals to analyze the regeneration process of skeletal muscle in FGF6 (-/-) mutant mice, but contradictory results emerged (Floss et al., 1997; Fiore et al., 1997; 2000; Armand et al., 2005). At present, the exact role of FGF6 in mouse muscle development and enlargement remains unclear (Armand et al., 2005). However, the FGF signaling pathway composed and its receptors FGFR1 (fibroblast growth factor receptor 1) and FGFR4 (fibroblast growth factor receptor 4) play important roles in the growth and development of muscle cells(muscle fibers). In the cellular experiment, muscle cells containing FGFR1 or FGFR4 were observed to alternately switch to different signaling pathways (Armand et al., 2006), whereas in vivo study indicated that both FGF6 and FGFR4 were exclusively expressed in myofibroblasts and astrocytes and FGFR1 was widely expressed in myogenic cells and all the cells (Kästner et al., 2000; Armand et al., 2005; Mcfarlane et al., 2006; Armand et al., 2006). In contrast, inhibition of FGFR4 signaling in vitro often led to the retention of muscle precursor cells' differentiation (Marics et al., 2002; Armand et al., 2005; Mcfarlane et al., 2006; Armand et al., 2006; Zipora et al., 2015). In addition to FGF6, many other FGFs are also expressed in skeletal muscles, including FGF2, FGF5 and FGF7. Most in vivo experiments have reported that these FGFs can induce the proliferation of myogenic cells and muscle satellite cells, whereas FGF1, FGF4, FGF2 and FGF6 can promote the cell proliferation of muscle astrocytes too (Marics et al., 2002; Armand et al., 2005; Mcfarlane et al., 2006; Armand et al., 2006; Zipora et al., 2015).

In another case of chicken muscles, FGFs regulated the differentiation of myogenic precursor cells, rather than alter the proliferative capacity of myogenic precursor cells (Duclos, 1998; Duclos et al., 1999; Duclos, 2005; Marics et al., 2002; Fisher et al., 2002). However, in reported studies, it is currently still unclear how do FGFs regulate the differentiation and myogenesis of myogenic precursor cells and muscle fibers. In the studies of zebrafish muscles, FGF8 and FGF24 work together during the developmental formation of mesoderm (Ochi and Westerfield, 2007). The cellular expression of FGF8 was detectable at the edge of the blastoderm during the $30 \%$ outsourcing period, whereas a back-abdominal gradient was formed during the $50 \%$ outsourcing period with the highest expression level observed on the dorsal side of the embryonic membrane. On the contrary, the earliest expression of FGF24 was detected at the edge of the blastoderm during approximately 50-60\% outsourcing period too (Ochi and Westerfield, 2007). During the period of zebrafish segmental segmentation, the expression of FGF8 extends to the late period of mesoderm before that of somite formation. FGF8 is also observed to be expressed in somites, whereas the expression of FGF24 is not detected in somites (Ochi and Westerfield, 2007). In the zebrafish FGF8 cerebellar mutant embryos, the expression of MyoD is reduced in the paraxial but remains unchanged in the somite, 
whereas the functional inhibition of FGF24 will suppress the formation of late mesodermin the FGF8 mutant embryos and the expression of MyoD was essentially removed and eliminated (Ochi and Westerfield, 2007), suggesting that FGF8 and FGF24 work together to regulate the formation of mesoderm and the gene expression of MyoD in zebrafish.

\section{Conclusion and Outlook}

At present, there are a considerable amount of studies and reports on the transcriptional regulation of animal myogenesis and muscle development (mostly skeletal muscles), but most of them are genetic and/or developmental analyses at the embryonic or cellular levels. There are lack of studies and/or reports on the gene expression networks and individual phenotypic effects. Furthermore, the research in depth on the myogenesis and development of muscle cells is presently not ample and elaborate enough. Particularly, the research on the roles of complex gene regulatory networks and underlying signaling pathways in myogenesis and muscle development is lack of systematic analyses. Although the framework of myogenesis and genetic network of skeletal muscle development has been proposed in the studies of zebrafish and fruit fly (Ochi and Westerfield, 2007; Haye et al., 2014), it is still only an ideal model and the specific studies and/or reports has not yet been fully carried out and reported.

In the future, if one is willing to conduct a comprehensive and systematic study on the relationship between myogenic regulatory genes' regulation and transcription factors' interaction during myogenesis and muscle development like genetic analyses in yeast, the high throughput sequencing technologies and modern genome level or genome-wide scale research techniques would be referred to simultaneously obtain a large number of biological data and bioinformation on the genetic expression levels of specific genes and their interactions. Fortunately, there are already such technologies, such as gene-chip or DNA microarray, SAGE (Serial Analysis of Gene Expression), MPSS (Massively Parallel Signature Sequencing), RNA-seq (RNA sequencing) and scRNA-seq (Single-cell RNAsequencing) and a series of analyzing techniques like bioinformatics and "Omics" techniques. Predictably, with the application of these biological technologies and/or techniques to the differential expression and analysis of specific genes involved in animal myogenesis and muscle development, the future research will provide a comprehensive and systematic information available for us, such as transcriptome and gene methylation. Of course, there will be more complete and systematic biological data and bioinformation enough for elaborated and in depth studies and/or reports on myogenesis and muscle development. This may be one of the promising directions of future research on the genetic regulation of animal muscle mass.

\section{Acknowledgment}

We are grateful to the anonymous reviewers for their constructive comments and suggestions. It is jointly funded by the Major Project of Municipal Horizontal Cooperation between Fuyang Government and Fuyang Normal University (No. XDHX201725) and the Major Project of Anhui Provincial Educational Commission Natural Science Foundation (No. KJ2016SD47) and the Project of National Natural Science Foundation of China (No.31301965).

\section{Ethics}

This is original and unpublished article. There are no animals dealt in the study and the author declared that no competing interests and no ethical issues involved. The corresponding author confirms that the author has read and approved the manuscript.

\section{References}

Aiello, D., K. Patel and E. Lasagna, 2018. The myostatin gene: An overview of mechanisms of action and its relevance to livestock animals. Anim Genet., 49: 505-519. DOI: 10.1111/age.12696

Alfonso, M.A. and S. Alison, 2002. Molecular Principles of Animal Development. 1st Edn., London: Oxford University Press, ISBN 0-19-879284-0.

Andersson, L. and M. Georges, 2004. Domestic-animal genomics: Deciphering the genetics of complex traits. Nature Rev. Genetics, 5: 202-212.

DOI: $10.1038 / \mathrm{nrg} 1294$

Armand, A.S., C. Pariset, I. Laziz, T. Launay and F. f et al., 2005. FGF6 regulates muscledifferentiation through a calcineurin-dependent pathway in regeneratingsoleus of adult mice. J. Cell. Physiol., 204: 297-308.

DOI: $10.1002 /$ jcp.20302

Armand, A.S., I. Laziz and C. Chanoine, 2006. FGF6 in myogenesis. Biochimica et Biophysica Acta, 1763: 773-778. DOI: 10.1016/j.bbamcr.2006.06.005

Atchley, W.R .and W.M. Fitch, 1997. A natural classification of the basic helix-loop-helix class of transcription factors. Proc. Nat. Acad Sci. USA, 7: 5172-5176. DOI: 10.1073/pnas.94.10.5172

Benezra, R., R.L. Davis, D. Lockshon, D.L. Turner and H. Weintraub, 1990. The protein Id: A negative regulator of helix-loop-helix DNA-binding proteins. Cell, 61: 49-59.

DOI: $10.1016 / 0092-8674(90) 90214-Y$ 
Berkes, C.A. and S.J. Tapscott, 2005. MyoD and the transcriptional control of myogenesis. Seminars Cell Developmental Biology, 16: 585-595. DOI: 10.1016/j.semcdb.2005.07.006

Black, B.L., J.D. Molkentin and E.N. Olson, 1998. Multiple roles for the MyoD basic region in transmission of transcriptional activation signals and interaction with MEF2. Mol. Cell Biol., 18: 69-77. DOI: $10.1128 / \mathrm{mcb} .18 .1 .69$

Brennan, T.J., T. Chakraborty and E.N. Olson, 1991. Mutagenesis of themyogenin basic region identifies an ancient protein motif criticalfor activation of myogenesis. Proc. Natl. Acad Sci. USA, 88: 5675-5679. DOI: 10.2307/2357241

Chelh, I., B. Meunier, B. Picard and M.J. Reecy, 2009. Molecular profiles of Quadriceps muscle inmyostatin-null mice reveal PI3K and apoptotic pathways as myostatin targets. Bmc. Genomics, 10: 196-0. DOI: 10.1186/1471-2164-10-196

Chen, A.C., N. Kraut, M. Groudine and H. Weintraub, 1996. I-mf, a novel myogenicrepressor, interacts with members of the MyoD family. Cell, 86: 731-741. DOI: 10.1016/S0092-8674(00)80148-8

Choi, J., M.L. Costa, C.S. Mermelstein and C. Chagas, 1990. Myod converts primary dermal fibroblasts, chondroblasts, smooth muscle and retinal pigmented epithelial cells into striated mononucleated myoblasts and multinucleated myotubes. Proc. Natl. Acad Sci. USA, 87: 7988-7992.

DOI: $10.1073 /$ pnas.87.20.7988

Cossu, G. and S. Biressi, 2005. Satellite cells, myoblasts and other occasional myogenic progenitors: Possible origin, phenotypic features and role in muscle regeneration. Seminars Cell Developmental Biology, 16: 623-631. DOI: 10.1016/j.semcdb.2005.07.003

Dichoso, D., T. Brodigan, K.Y. Chwoe and J.S. Lee, 2000. The MADS-box factor CeMEF2 is not essential for caenorhabditis elegans Myogenesis and development. Developmental Biol., 223: 431-440. DOI: $10.1006 /$ dbio. 2000.9758

Dominique, J.E. and G. Cabello, 2006. Myostatin regulation of muscle development: Molecular basis, natural mutations, physio-pathological aspects. Experimental Cell Res., 312: 2401-2414. DOI: $10.1016 /$ j.yexcr.2006.04.012

Dou, T., Z. Li, K. Wang and L. Liu, 2018. Regulation of myostatin expression is associated with growth and muscle development in commercial broiler and DMC muscle. Mol. Biol. Rep., 45: 511-522. DOI: $10.1007 / \mathrm{s} 11033-018-4187-7$

Duclos, M.J., 1998. Regulation of chicken muscle growth by insulin-like growth factors. Trends in comparative endocrinology and neurobiology. Ann. NY Acad Sci., 839: 166 -171.

DOI: $10.1111 /$ j.1749-6632.1998.tb10752.x
Duclos, M.J., 2005. Insulin-like growth factor-I (IGF-1) mRNA levels and chicken muscle growth. J. Physiology Pharmacology, 56: 25-35.

PMID: 16077194

Duclos, M.J., C. Beccavina and J. Simona, 1999. Genetic models for the study of Insulin-like Growth Factors (IGF) and muscle development in birds compared to mammals. Domestic Anim. Endocrinology, 17: 231-243.

DOI: 10.1016/S0739-7240(99)00040-5

Edmondson, D.G. and E.N. Olson, 1993. Helix-loophelix proteins as regulators of muscle-specific transcription. J. Biological Chemistry, 268: 755-758. PMID: 8380414

Fiore, F., A. Sebille and D. Birnbaum, 2000. Skeletal muscle regeneration is notimpaired in Fgf6-/mutant mice. Biochem. Biophys. Res. Commun., 272: 138-143. DOI: 10.1006/bbrc.2000.2703

Fiore, F., J. Planche, P. Gibier, A. Sebille and O. de Lapeyriere et al., 1997. Apparent normal phenotype of Fgf6-/- mice. Int. J. Dev. Biol., 41: 639-642. PMID: 9303352

Fisher, M.E., H.V. Isaacs and M.E. Pownall, 2002. eFGF is required for activation of $\mathrm{XmyoD}$ expression in the myogenic celllineage of Xenopus laevis. Development, 129: 1307-1315.

DOI: $10.1068 / \mathrm{a} 36295$

Floss, T., H.H. Arnold and T. Braun, 1997. A role for FGF6 in skeletal muscle regeneration. Genes Dev., 11: 2040-2051. DOI: 10.1101/gad.11.16.2040

Franck, V. and A. Mauviel, 2002. Transforming growth factor-beta signaling through the Smad pathway: Role in extracellular matrix gene expression and regulation. J. Invest Dermatol., 118: 211-215. DOI: $10.1046 /$ j.1523-1747.2002.01641.x

Franck, V., M.L. Chu and A. Mauviel, 2001. Identification of novel TGF-beta /Smad gene targets in dermal fibroblasts using a combined cDNA microarray/promoter transactivation approach. J. Biol. Chem., 276: 17058-17062.

DOI: $10.1074 /$ jbc.M100754200

Georges, M., 2007. Mapping, fine mapping and molecular dissection of quantitative trait loci in domestic animals. Ann. Review Genomics Human Genetics, 8: 131-162.

DOI: 10.1146/annurev.genom.8.080706.092408

Hamamori, Y., H.Y. Wu, V. Sartorelli and L. Kedes, 1997. The basic domain of myogenic basic helixloop-helix (bHLH) proteins is the novel target for direct inhibition by another bHLH protein Twist. Mol. Cell Biol., 17: 6563-73. DOI: $10.1128 / \mathrm{mcb} .17 .11 .6563$

Haye, A., J. Albert and M. Rooman, 2014. Modeling the drosophila gene cluster regulation network for muscle development. PLOS ONE, 9: e90285. DOI: 10.1371/journal.pone.0090285 
Headey, S.J., D.W. Keizer, S. Yao, J.C. Wallace and L.A. Bach et al., 2004. Binding site for the Cdomain of insulin-like growth factor (IGF) binding protein-6 on IGF-II: Implications for inhibition of IGF actions. FEBS Lett., 568: 19-22.

DOI: 10.1016/j.febslet.2004.04.091

Heanue, T.A., R. Reshef, R.J. Davis and G. Mardon, 1999. Synergistic regulation of vertebrate muscle development by dach2, eya2 and six1, homologs of genes required for drosophila eye formation. Genes Development, 13: 3231-3243.

DOI: $10.1101 / \mathrm{gad} .13 .24 .3231$

Heldin, C.H., K. Miyazono and P. Dejke, 1997. TGF- $\beta$ singling from cell membrane to nucleus through SMAD proteins. Nature, 390: 465-472.

DOI: $10.1038 / 37284$

Hitachi, K. and K. Tsuchida, 2017. Myostatin-deficiency in mice increases global gene expression at the Dlk1-Dio3 locus in the skeletal muscle. Oncotarget, 8: 5943-5953. DOI: 10.18632/oncotarget.13966

Huang, J., H. Weintraub and L. Kedes, 1998. Intramolecular regulation of MyoD activation domain conformation and function. Mol. Cell Biol., 18: 5478-84. DOI: $10.1128 / \mathrm{mcb} \cdot 18.9 .5478$

Itoh, N. and D.M. Ornitz, 2011. Fibroblast growth factors: from molecular evolution to roles in development, metabolism and disease. J. Biochem., 149: 121-130. DOI: $10.1093 / \mathrm{jb} / \mathrm{mvq} 121$

Kablar, B., A. Asakura, K. Krastel, C. Ying and L.L. May et al., 1998. MyoD and Myf-5 define the specification of musculature of distinct embryonic origin. Biochem. Cell Biol., 76: 1079-1091.

DOI: 10.1139/bcb-76-6-1079

Kassarduchossoy, L., B. Gayraudmorel, D. Gomès and D. Rocancourt, 2004. Mrf4 determines skeletal muscle identity in Myf5:Myod double-mutant mice. Nature, 431: 466-471. DOI: 10.1038/nature02876

Kästner, S., M.C. Elias, A.J. Rivera and Z. YablonkaReuveni, 2000. Gene expression patterns of the fibroblast growth factors and their receptors during myogenesis of rat satellite cells. J. Histochem. Cytochem., 48: 1079-1096.

DOI: $10.1177 / 002215540004800805$

Kawada, S., C. Tachi and N. Ishii, 2001. Content and localization of myostatin in mouse skeletal muscles during aging, mechanical unloading and reloading. J. Muscle Res. Cell Motil., 22: 627-33. DOI: 10.1023/A:1016366409691

Lemercier, C., R.Q. To, R.A. Carrasco and S.F. Konieczny, 1998. The basic helixloop-helix transcription factor Mist1 functions as a transcriptional repressor of MyoD. EMBO J., 17: 1412-1422. DOI: 10.1093/emboj/17.5.1412

Lessard, S.J., T.L. MacDonald, P. Pathak and M.S. Han, 2018. JNK regulates muscle remodeling via myostatin/SMAD inhibition. Nat. Commun., 9: 3030. DOI: $10.1038 / \mathrm{s} 41467-018-05439-3$
Lin, Q., J. Schwarz and E.N. Bucana Cand Olson, 1997. Control of mouse cardiac morphogenesis and myogenesis by transcription factor MEF2C. Science, 276: 1404-1407.

DOI: $10.1126 /$ science. 276.5317 .1404

Lu, J., R. Webb, J.A. Richardson and E.N. Olson, 1999. MyoR: A muscle restricted basic helix-loop-helix transcription factor that antagonizes the actions of MyoD. Proc. Natl. Acad Sci. USA, 96: 552-7. DOI: $10.2307 / 46849$

Maqbool, T. and K. Jagla, 2007. Genetic control of muscle development: Learning fromdrosophila. J. Muscle Res. Cell Motility, 28: 397-407. DOI: 10.1007/s10974-008-9133-1

Marics, I., F. Padilla, J.F. Guillemot, M. Scaal and C. Marcelle, 2002. FGFR4 signaling is a necessary step in limb muscle differentiation. Development, 129: 4559-4569. DOI: $10.1007 / \mathrm{s} 00429-002-0268-3$

Mcfarlane, C., E. Plummer, M. Thomas and A. Hennebry, 2006. Myostatin induces cachexia by activating the ubiquitin proteolytic system through an NF-kappaB-independent, FoxO1-dependent mechanism. J. Cellular Physiol., 209: 501-514. DOI: $10.1002 /$ jcp.20757

McMurtry, J.P., G.L. Francis and Z. Upton, 1997. Insulin-like growth factors in poultry. Domest Anim. Endocrinol., 14: 199-229. DOI: 10.1016/S0739-7240(97)00019-2

McPherron, A.C., A.M. Lawler and S.J. Lee, 1997. Regulation of skeletal muscle mass in mice by a new TGF- $\beta$ super-family member. Nature, 387: 83-90. DOI: $10.1038 / 387083 \mathrm{a} 0$

Molkentin, J.D. and E.N. Olson, 1996. Defining the regulatory networks for muscle development. Current Opinion Genetics Development, 6: 445-453. DOI: $10.1016 / \mathrm{S} 0959-437 \mathrm{X}(96) 80066-9$

Murre, C., P.S. Mccaw, H. Vaessin and M. Caudy, 1989. Interactions between heterologous helix-loop-helix proteins generate complexes that bind specifically to a common DNA sequence. Cell, 58: 537-544. DOI: 10.1016/0092-8674(89)90434-0

Myer, A., E.N. Olson and W.H. Klein, 2001. MyoD cannot compensate for the absence of myogenin during skeletal muscle differentiation inmurine embryonic stem cells. Dev. Biol., 229: 340-50. DOI: $10.1006 /$ dbio.2000.9985

Mynarcik, D.C., G.Q. Yu and J. Whittaker, 1996. Alanine-scanning mutagenesis of a C-terminal ligand binding domain of the insulin receptor alpha subunit. J. Biol. Chem., 271: 2439-2442.

DOI: $10.1074 /$ jbc.271.5.2439

Nabeshima, Y., 1996. [gene network regulating muscle development]. Tanpakushitsu Kakusan Koso Protein Nucleic Acid Enzyme, 41: 1008-1017. PMID: 8741620 
Neuhold, L.A. and B. Wold, 1993. HLH forced dimers: Tethering MyoD to E47 generates a dominant positive myogenic factor insulated from negative regulation by Id. Cell, 74: 1033-1042. DOI: $10.1016 / 0092-8674(93) 90725-6$

Ochi, H. and M. Westerfield, 2007. Signaling networks that regulate muscle development: lessons from zebrafish. Development Growth Differentiation, 49: 1-11. DOI: 10.1111/j.1440-169X.2007.00905.x

Odle, J., S.K. Jacobi, R.D. Boyd, D.E. Bauman and R.V. Anthony et al., 2017. The potential impact of animal science research on global maternal and child nutrition and health: A landscape review. Advances Nutrition: An Int. Rev. J., 8: 362-381.

DOI: 10.3945/an.116.013896

Ornitz, D.M. and N. Itoh, 2001. Fibroblast growth factors, Genome Biol., 2: REVIEWS3005REVIEWS3012. PMID: 11276432

Palacios, D. and P.L. Puri, 2006. The epigenetic network regulating muscle development and regeneration. J. Cellular Physiology, 207: 1-11.

DOI: $10.1002 /$ jcp.20489

Parrinello, S., C.Q. Lin, K. Murata, Y. Itahana and J. Singh et al., 2001. Id-1, ITF-2 and Id-2 comprise a network of helix-loop-helix proteins that regulate mammary epithelial cell proliferation, differentiation and apoptosis. J. Biological Chemistry, 276: 39213-39219. DOI: $10.1074 /$ jbc.M104473200

Penn, B.H., D.A. Bergstrom, F.J. Dilworth, E. Bengal and S.J. Tapscott, 2004. A MyoD-generated feedforward circuit temporally patterns gene expression during skeletal muscle differentiation. Genes Dev., 18: 2348-2353. DOI: 10.1101/gad.1234304

Pownall, M.E., M.K. Gustafsson and C.P.J. Emerson, 2002. Myogenic regulatory factors and the specification of muscle progenitors in vertebrate embryos. Annu. Rev. Cell Dev. Biol., 18: 747-783. DOI: $10.1016 /$ j.amjcard.2004.06.028

Rawls, A. and E.N. Olson, 1997. MyoD meets its maker. Cell, 89: 5-8. DOI: 10.1016/S0092-8674(00)80175-0

Rawls, A., J.H. Morris, M. Rudnicki and T. Braun, 1995. Myogenin's Functions Do Not Overlap with Those of MyoD or Myf-5 during Mouse Embryogenesis. Developmental Biology, 172: 37-50.

DOI: $10.1006 /$ dbio. 1995.0004

Robson, L.G., 2017. Vertebrate embryo: Myogenesis and muscle development. eLS, John Wiley \& Sons, Ltd.

Rossi, G., S. Antonini, C. Bonfanti and S. Monteverde, 2016. Nfix regulates temporal progression of muscle regeneration through modulation of Myostatin expression. Cell Reports, 14: 2238-2249.

DOI: 10.1016/j.celrep.2016.02.014
Rudnicki, M.A., P.N.J. Schnegelsberg, R.H. Stead and T. Braun, 1993. MyoD or Myf-5 is required for the formation of skeletal muscle. Cell, 75: 1351-1359. DOI: 10.1016/0092-8674(93)90621-V

Spicer, D.B., J. Rhee, W.L. Cheung and A.B. Lassar, 1996. Inhibition of myogenic bHLH and MEF2 transcription factors by the bHLH protein Twist. Science, 272: 1476-80.

DOI: $10.1126 /$ science.272.5267.1476

Sumariwalla, V.M. and W.H. Klein, 2001. Similar myogenic functions for myogenin and MRF4 but not MyoD in differentiated murine embryonic stem cells. Genesis, 30: 239-249.

DOI: $10.1002 /$ gene. 1080

Szebenyi, G. and J.F. Fallon, 1999. Fibroblast growth factors as multifunctional signaling factors. Int. Rev. Cytol., 185: 45-106.

DOI: $10.1016 / \mathrm{S} 0074-7696(08) 60149-7$

Tajbakhsh, S., D. Rocancourt, G. Cossu and M. Buckingham, 1997. Redefining the genetic hierarchies controlling skeletal myogenesis: Pax-3 and Myf-5 act upstream of MyoD. Cell, 89: 127-138. DOI: 10.1016/S0092-8674(00)80189-0

Wang, Y. and R. Jaenisch, 1997. Myogenin can substitute for Myf5 in promoting myogenesis but less efficiently. Development, 124: 2507-13. DOI: $10.1007 / \mathrm{s} 004290050081$

Whittaker, J., H. Sorensen, V.L. Gadsboll and J. Hinrichsen, 2002. Comparison ofthe functional insulin binding epitopes of the A and B isoforms of the insulin receptor. J. Biol. Chem., 277: 4738047384. DOI: $10.1074 /$ jbc.M208371200

Wilson-Rawls, J., J.D. Molkentin, B.L. Black and E.N. Olson, 1999. Activated notch inhibits myogenic activity of the MADS-box transcription factor myocyte enhancer factor 2C. Molecular Cellular Biology, 19: 2853-2862.

DOI: $10.1128 / \mathrm{mcb} \cdot 19.4 .2853$

Zhu, Z. and J.B. Miller, 1997. MRF4 can substitute for myogenin during earlystages of myogenesis. Dev Dyn., 209: 233-41. DOI: 10.1002/(SICI)10970177(199706)209:2<233::AID-AJA9>3.0.CO;2-J

Zipora, Y.R., M.E. Danoviz, P. Michael and S. Pascal, 2015. Myogenic-specific ablation of FGFrl impairs FGF2-mediated proliferation of satellite cells at the myofiber niche but does not abolish the capacity for muscle regeneration. Front Aging Neurosci., 7: 85. DOI: $10.3389 /$ fnagi.2015.00085a 